Original Paper http://ajol.info/index.php/ijbcs http://indexmedicus.afro.who.int

\title{
Persistance de la schistosomiase urinaire en zones endémiques soumises aux traitements de masse répétés au Mali
}

\author{
Bintou LY ${ }^{1}$, Alpha Seydou YARO ${ }^{1,3^{*}}$, Bernard SODIO ${ }^{1}$ et Moussa SACKO ${ }^{2}$ \\ ${ }^{1}$ Faculté des Sciences et Techniques, Université des Sciences des Techniques et des Technologies de Bamako \\ FST ; USTTB ; BP 3206 Bamako, Mali. \\ ${ }^{2}$ Institut National de Recherche en Santé Publique (INRSP) ; BP 1771 Bamako, Mali. \\ ${ }^{3}$ International Centre for Research Excellency in Mali (ICER-Mali); Faculté de Médecine et \\ d'Odontostomatologie FMOS ; Point G ; BP 1805 ; USTTB, Bamako, Mali. Centre Collaborateur du NIH- \\ NIAID USA. \\ *Auteur correspondant ; E-mail : alphaseydouyaro@gmail.com,yaro@icermali.org
}

\section{REMERCIEMENTS}

Les auteurs de ce travail remercient le PPF (Programme de Formation des Formateurs) de Bamako pour avoir financé les activités de terrains de cette étude dans le cadre de la formation doctorale de Bintou LY, Enseignant-chercheur, Assistant au DER de Biologie de la FST-USTTB.

\section{RESUME}

Au Mali, la schistosomiase est un problème de santé publique comme dans tous les 42 pays africains sur 76 concernés dans le Monde, avec 230 millions de personnes infectées sur 800 millions de personnes exposées et plus de 800000 décès annuels. L'objectif de cette étude était de comprendre les complications de la schistosomiase par des investigations parasitologue, échographique et clinique dans trois zones endémiques à traitement de masse répété de la région de Ségou au Mali. Les sites d'étude : Guénidanga, Kalabougou et M'Pèba sont situés le long du fleuve Niger. Le diagnostic était basé sur la filtration de l'urine, le KATO-KATZ des selles, l'examen physique, la réplétion vésicale et l'échographie abdominopelvienne. Sur les 327 sujets de l'étude, $50,80 \%$ d'individus infectés avaient au plus 5 ans suivie de ceux $\geq 24$ ans avec $22 \%$. La charge ovulaire était plus élevée chez les sujets de 5 à 14 ans. Malgré un traitement de masse répété, des complications ont engendré d'importantes lésions rénales et vésicales chez les sujets masculins. La persistance de la schistosomiase humaine pourrait être due soit à une probable réinfection rapide, soit à une fluctuation de la population des mollusques dans ces zones.

(C) 2019 International Formulae Group. All rights reserved.

Mots clés: Bilharziose urinaire, complication, lésion vésicales et rénales.

\section{Persistence of urinary schistosomiasis in endemic areas subjected to repeated mass treatments in Mali}

\begin{abstract}
Schistosomiasis is a real public health problem in endemic countries. Up to 42 of 76 concerned countries are in Africa. As second endemic disease after malaria, 230 million people are infected out of 800 million exposed people with more than 800,000 deaths annually. The objective of this study was to understand
\end{abstract}


complications due to schistosomiasis by parasitological, echographical and clinical investigations in three endemic zones involved in repeated mass treatment in Ségou, Mali. The study sites are located along the Niger River in Ségou, Mali. The study population was composed by 327 subjects leaving in the villages of Guénidanga, Kalabougou and M'Pèba. Results show that young subjects less than 5 years old representing $50.80 \%$ are more infected; Followed by the age group greater than $24(22 \%)$. Ovular load was higher in subjects from 5 to 14 years old. Although people were under repeated mass treatment, cases of complications were recorded. Prevalence infection analyses among subjects show no sex dependent. Renal and vesical lesions were higher in male gender than female. Either this could be due to a probable reinfection or to a fluctuation of interme-diate host population in those villages.

(C) 2019 International Formulae Group. All rights reserved.

Keywords: Urinary bilharziasis, complication, vesical and renal lesions.

\section{INTRODUCTION}

La schistosomiase est une maladie parasitaire eau dépendante très répandue dans le monde, surtout en Afrique sub-saharienne (OMS, 2016). Jusqu'en 2011, la Bilharziose sévissait encore dans 76 pays situés en Afrique, en Amérique du sud, au Moyen Orient et au Sud du continent asiatique (WHO, 2011). Deuxième endémie après le paludisme dans les régions tropicales et subtropicales (Engels et al., 2002; WHO, 2011), la schistosomiase ou bilharziose constitue un véritable problème de santé publique dans les pays endémiques dont 42 pays en Afrique (Traore et al., 2007 : Clements et al., 2008 ; OMS, 2010). En effet certaines études ont montrés que près de 800 millions de personnes y sont exposées dans le monde (Aubry, 2016). Sur 230 millions de personnes infectées subissant le traitement annuel, 80 à $90 \%$ vivent en Afrique avec 800000 décès par an (Aubry, 2016), dont 280000 cas de décès dus aux complications (Chippaux, 2000 ; Chitsulo et al., 2004 ; WHO, 2011).

Au sud du Sahara, elle est la cause d'une hématurie et d'atteinte grave des parois chez des millions de personnes. La zone de prévalence se situe dans les régions tropicales et subtropicales des communautés vivant le long des cours d'eau. Beaucoup d'études ont confirmé que la répartition des schistosomiases était fortement liée à l'environnement physique. Par exemple, les aménagements hydro agricoles créent un contexte favorable au développement de cette maladie (Molyneux et al., 2005 ; Steinman et al., 2006; Garcia et al., 2013 ; Yapi et al., 2017). La co infection entre -S. hæematobium, $S$. mansoni ou bien entre $S$. hamatobium et $S$. gueneesis (Moné et al., 2012) a été déjà documentée dans certains pays d'Afrique subsaharienne.

Au Mali, la schistosomiase a fait l'objet de beaucoup d'études sur l'épidémiologie (Tandina et al., 2016), la variabilité génétique, le degré de l'intensité de l'infection et enfin sur la morbidité ; surtout dans les zones endémique. La Schistosomiase est endémique dans de nombreuses régions du pays (Traore et al., 2007 ; Clements et al., 2008). Mais les niveaux de transmission sont très variables d'une région à une autre et d'un village à un autre dans la même zone bioclimatique (Traore et al., 2007). Présentement, le Mali a un taux de prévalence global de $30 \%$ avec une différence entre les différentes zones endémiques. Les zones hyper endémiques qui sont les terres irriguées de l'Office du Niger à Ségou, le plateau Dogon avec des petits barrages, le bassin du fleuve Sénégal ont une prévalence supérieure à 50\% Steinman (2006). Les zones soudaniennes et nord soudaniennes sont moins touchées avec une prévalence de 10\% (Traore 
et al., 2007). Actuellement dans la région de Ségou des études ont données une prévalence de $79,8 \%$ pour $S$. haematobium et $13,2 \%$ pour S. mansoni (Stecher et al., 2017).

Selon l'OMS l'infection de la forme urinaire a entrainée chez $1 / 3$ des enfants de 7 à 14 ans une dilatation urétérale et chez $1 / 2$, des lésions de la vessie. Aussi, à travers des études faites en 2001 au service d'urologie de l'hôpital du Point G, il a été révélé que les complications dues à la bilharziose urinaire constituent la troisième cause des interventions chirurgicales de ce service (Tangara, 2002). La chimiothérapie a été la stratégie de choix de l'OMS à travers les programmes nationaux de lutte contre la schistosomiase pour la prévention et la réduction de la morbidité. Ce programme existe au Mali depuis 1982; il est axé principalement sur le traitement de masse au praziquantel dans la zone de l'Office du Niger et à Bandiagara. Actuellement le traitement de masse avec le praziquantel au sein de la population à risque (les enfants d'âge préscolaires et scolaire, les pêcheurs, agriculteurs les femmes etc.) demeure l'approche principale ayant le meilleur rapport coût /efficacité, la plus faisable et la plus durable. Malgré ces traitements annuels ou biannuels, il a été rapporté que la réinfection survient dans les zones endémiques en moins de 12 mois chez plusieurs enfants traités (N'Goran et al., 2001). La succession d'épisodes d'infection et de réinfection ainsi qu'un refus du traitement provoque une chronicité de l'infection dans les zones endémiques. De nos jours encore, les complications dues à S. harmatobium sont mal connues. C'est dans cette dynamique que cette étude a été entreprise afin de générer des résultats scientifiques pouvant convaincre les décideurs à percevoir d'avantage la portée de la bilharziose urinaire pour l'amélioration de la prise en charge des malades et la conduite des traitements de masse dans les villages endémiques situées au bord du fleuve Niger au Mali. Ces résultats pourront éclairer le programme national de lutte contre cette infection à revoir sa stratégie.

\section{MATERIEL ET METHODES}

\section{Sites d'étude}

Cette étude s'est déroulée dans 3 localités situées au bord du fleuve Niger dans la région de Ségou: Guénidanga, Kalabougou, et M'Pèba (Figure 1). Ségou est la $4{ }^{\text {ème }}$ région administrative du Mali et fait frontière avec toutes les régions du Mali à l'exception de Kayes, Gao et Kidal. Elle a une superficie de $60947 \mathrm{~km}^{2}$, soit environ 5\% du territoire Malien. Elle a une économie fortement liée à l'agriculture pratiquée par $80 \%$ de la population (Zone Office du Niger). Elle est divisée en 118 communes dont 3 urbaines réparties entre 7 cercles. Le climat est de type Soudano-sahélien. Les hauteurs de pluies varient entre 400 et $800 \mathrm{~mm}$ par an. Le relief est peu accidenté, on y trouve des formations arbustives, épineuses et la savane arborée. Le réseau hydrographique de la région de Ségou est l'un des meilleurs du pays au regard des potentialités agro-pastorales. Il est constitué principalement par le fleuve Niger et son affluent, le Baní. Le Niger traverse Ségou sur $292 \mathrm{Km}$ et alimente un réseau d'irrigation grâce au barrage de Markala à travers trois canaux dont les eaux sont exploitées par l'Office du Niger pour le développement de la riziculture avec une maitrise totale de l'eau. L'économie de la région est basée principalement sur le secteur primaire, l'agriculture, et d'autres activités régénératrices à savoir, le commerce des ressources naturelles, le tourisme, la pêche et l'artisanat. Plus de $50 \%$ de la population est localisée presque au bord des cours d'eau. Les 
professions de contact avec l'eau sont très importantes ainsi que les activités quotidiennes : les activités domestiques et les baignades.

Il s'agit là d'une étude transversale prospective effectuée pendant le mois de juin 2013. L'échantillonnage était constitué de sujets jeunes, adultes (sous traitements de masse de l'INRSP depuis plus de 5 ans).

Pour être inclus dans l'étude, il fallait être un sujet sous traitement de masse. Avoir au moins 3 ans ; être résident d'un des villages d'étude et être disponible pour l'étude puis donner/signer un consentement éclairé (ou l'assentiment des parents le cas échéant).

N'ont pas été inclus dans l'étude, les sujets non disponibles ou handicapés et n'ayant pas donné leur consentement.

La charge d'excrétion ovulaire a été définie par le nombre total d'œufs comptés dans les $10 \mathrm{ml}$ d'urine filtrée. Selon la quantité d'œufs observés, les résultats peuvent être classés en trois catégories selon l'OMS :

Mesure de l'intensité de Schistosoma hormatobium :

0 Euf---------------- Non infesté

1-49 œufs/10 ml d'urine------------Faiblement Infesté

$\geq 50$ œufs / $10 \mathrm{ml}$ d'urine -------Fortement infesté

Le travail était organisé en quatre postes: *Un poste de recensement qui s'occupait de l'identification, la numérotation des volontaires de l'étude et la remise de bocal (blanc) pour les urines et un sachet noir pour les selles;*Un poste de parasitologie pour la récupération et le traitement de l'urine par filtration et les selles par la technique de KATO-KATZ ; *un poste Clinique pour avoir les paramètres cliniques et l'examen physique; et *un poste d'échographie permettant d'obtenir la réplétion vésicale et l'échographie abdominopelvienne.

\section{Les considérations éthiques :}

Le protocole de l'étude a été approuvé par le comité d'éthique de l'INRSP. La population des zones d'étude a reçu des informations claires et détaillées sur l'étude (les objectifs, la méthodologie et les avantages) avant la mise en exécution. La participation et le retrait à l'étude était volontaire. Le consentement éclairé des sujets majeurs et l'assentiment des parents des sujets mineurs ont été obtenu. La confidentialité et le respect de l'intérêt des sujets ont été pris en compte. Toutes les populations d'étude étaient systématiquement traitées par le praziquantel après la remise des échantillons.

\section{Analyse des données}

Les données étaient initialement enregistrées sur des fiches de collectes sur le terrain. Elles ont été saisies et enregistrées sur le logiciel Excel de Windows 2010. Un contrôle d'élimination de toutes les incohérences a été effectué avant la saisie. Les données ont ensuite été validées par l'utilisation de la technique de vérification aléatoire des entrées. Les résultats ont été présentés sous forme narrative, de tableaux, d'histogramme ou de diagramme en barres. La conversion du fichier Excel dans le logiciel SPSS.16.0 a permis de faire une première analyse qui a été complétée grâce au logiciel EpiInfo.6.0. La comparaison des effectifs entre village, sexe, et tranche d'âge a été faite par le «t» test. La comparaison des prévalences a été faite par le test de Khi carré soit de Fisher ou de Pearson en fonction de la convenance des analyses et le seuil de $5 \%$ a été considérée comme significatif. 


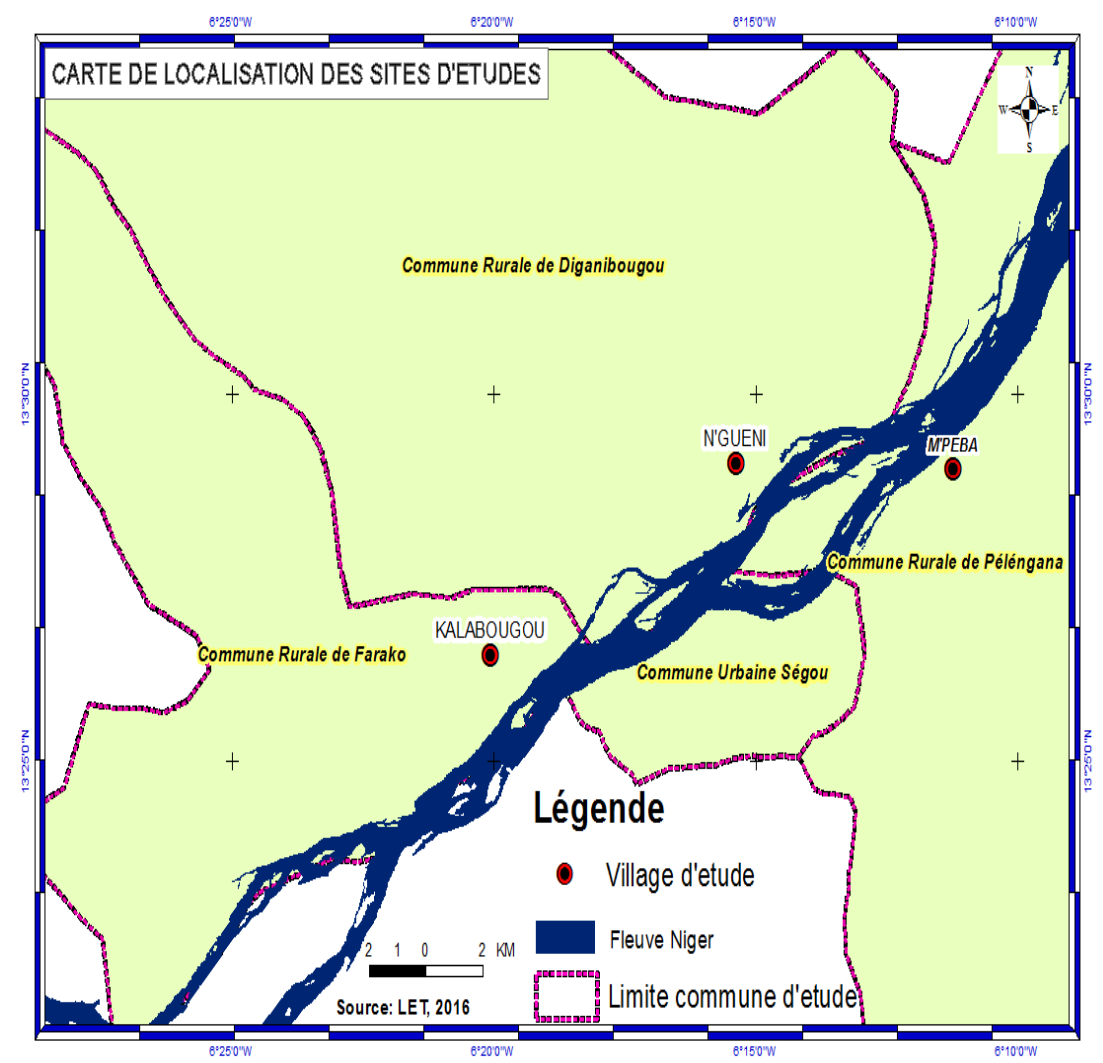

Figure 1 : Carte de la localisation des trois sites d'études : Kalabougou. N'Guénidanga et M'Pèba. NB : Guénidanga = N'GUENI ; Source : Unité SIG (Système d'Information Géographique du laboratoire d'écologie tropicale de la FST-USTTB. Mali.

\section{RESULTATS}

Dans cette étude nous n'avions pas trouvé des cas de S. mansoni.

\section{Données démographiques}

$\mathrm{Au}$ total 327 sujets résidents des villages de N'Guénidanga (110), Kalabougou (65) et M'Pèba (152) ont participé à cette étude. Les sujets ont été répartis en 4 tranches d'âge : $\leq 5 ;] 5-14] ; \quad[14-24]$ et $\geq 24$ (Tableau 1).

Sur un total de 327 sujets inclus, 152 individus étaient du village de M'Pèba, 110 sujets étaient de Guénidanga et 65 sujets de Kalabougou. Dans l'échantillonnage de cette étude, le nombre de sujets de sexe féminin $(58,10 \%)$ était supérieur à celui des sujets masculins $(41,90 \%): \mathrm{Chi}^{2}=8,45 ; \mathrm{ddl}=1 ; \mathrm{P}=$
0,003 avec un ratio de 1,39 en faveur du sexe féminin (Tableau 1). Cette même tendance de supériorité numérique des sujets féminins par rapport aux sujets masculins est observée dans chacun des trois sites d'études (Tableau 1).

Les sujets de la tranche d'âge de 5 à 14 ans représentent $50,8 \%$ des sujets et sont majoritairement originaire du village de M'Pèba (88/166) (Tableau 2). Par contre aucun sujet de plus de 24 ans n'a participé à l'étude dans ce village. Les sujets âgés de plus de 24 ans étaient beaucoup plus recensés dans le village de Guénidanga (32/48). Environ 78 $\%(255 / 327)$ des sujets étaient âgés d'au plus 24 ans. Sur les 327 sujets, 60 avaient l'âge inferieur ou égale à 5 ans; 16 avaient l'âge compris entre 5 et 14 ans ; 29 étaient âgés de 14 à 24 ans et 72 individus avaient plus de 24 ans (Tableau 2). 


\section{Parasitémie}

Parmi les sujets, 131 avaient une faible parasitémie, 16 sujets avaient une parasitémie moyenne et 35 sujets avaient une forte parasitémie (Tableau 3). Le rapport de sexe (Féminin/Masculin) par rapport à l'intensité de la charge ovulaire est de 79/52 ; $8 / 8$ et $20 / 15$ respectivement pour les sujets ayant une charge ovulaire faible, moyenne et élevée. Les tests statistiques ont montré qu'il n'y a pas de différence significative entre les sujets de sexe différent par rapport à l'intensité de la charge ovulaire $\left(\mathrm{Chi}^{2}\right.$ de Pearson $=0,674 ; \mathrm{ddl}=2 ; \mathrm{P}=0,714$ ) (Tableau $3)$.

\section{Charge ovulaire}

Pour les trois catégories de parasitémie (forte, moyenne et faible), c'est la tranche d'âge de 5 à 14 ans qui a la plus forte proportion, soit $65,62 \% ; 68,75 \%$ et $55,88 \%$ respectivement pour les groupes de charge ovulaire faible, moyenne et forte (Figure 2) . Dans le groupe de faible charge ovulaire (où les effectifs permettent une analyse statistique) il y a une différence significative entre charges ovulaires des différentes tranches d'âges $\left(\mathrm{Chi}^{2}=14,03 ; \mathrm{ddl}=3 ; \mathrm{P}=\right.$ $0,002)$. Une charge ovulaire élevée était pratiquement inexistante ou très faible chez les sujets de plus de 14 ans (Figure 2).

\section{Prévalence}

La prévalence de l'infection à $S$. haematobium dans le village de M'Pèba $(72,36 \%)$ est nettement supérieur à celles des villages de Guénidanga (52,61\%) et de Kalabougou (24,4\%) (Tableau 4). Cette différence était significative : $\mathrm{Chi}^{2}$ de Pearson $=43,04 ; \mathrm{ddl}=2 ; \mathrm{P}<10^{-6}$ (Tableau 4). Pour tous les villages confondus, la prévalence de l'infection à $S$. haematobium entre sujets de sexe différent n'était pas significativement différente (Tableau 5). La prévalence de l'infection au sein du groupe des sujets de sexe féminin $(57,84 \%)$ était comparable à celle au sein du groupe des sujets de sexe masculin $(55,73 \%): \mathrm{Chi}^{2}$ de Pearson $=0,19$; $\mathrm{ddl}=1 ; \mathrm{P}=0,662$ (Tableau 5)

\section{Données échographiques}

La proportion des sujets ayant une lésion vésicale $42,51 \% \quad(139 / 327) \quad$ est statistiquement supérieure à celle ayant une lésion rénale $3,67 \%$ (12/327): $\mathrm{Chi}^{2}$ de Pearson $=138,88 ; \mathrm{ddl}=1 ; \mathrm{P}<10^{-6}$ (Figure 3). Une comparaison entre volontaires féminins et masculins des prévalences de la lésion aussi bien vésicale $\left(\mathrm{Chi}^{2}=0,16 ; \mathrm{ddl}=1 ; \mathrm{P}=0,68\right)$ que rénale $\left(\mathrm{Chi}^{2}=1,46 ; \mathrm{ddl}=1 ; \mathrm{P}=0,226\right) \mathrm{a}$ montré qu'il n'y a pas de différence significative liée au sexe lorsqu'il s'agit du même type de lésion (Figure 4).

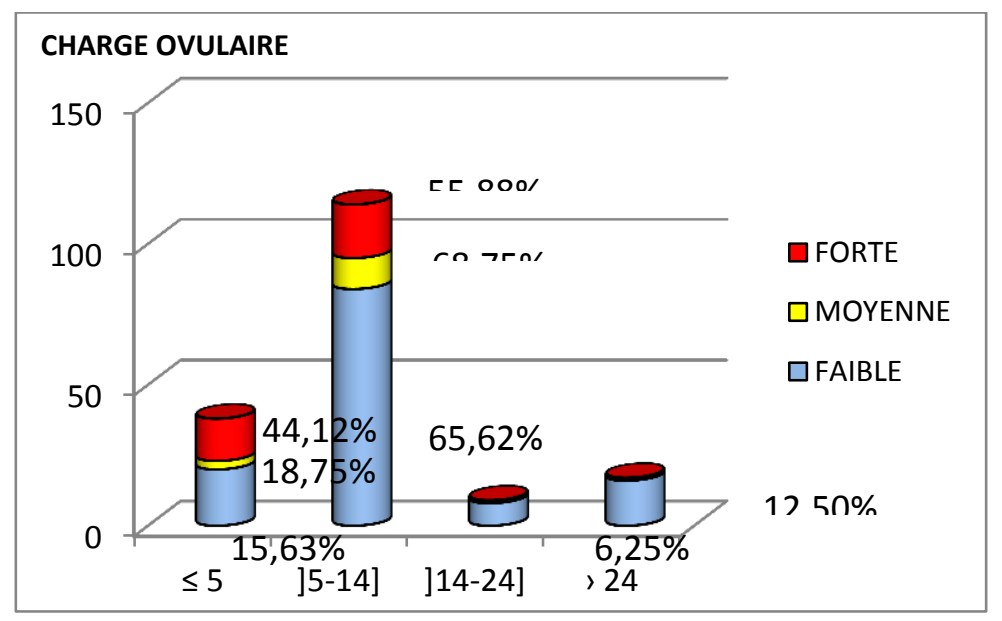

Figure 2 : Charge ovulaire des sujets de l'étude par tranche d'âge et en fonction de l'intensité de leur charge ovulaire pour $S$. haematobium. $\mathrm{P}=0.002$. 


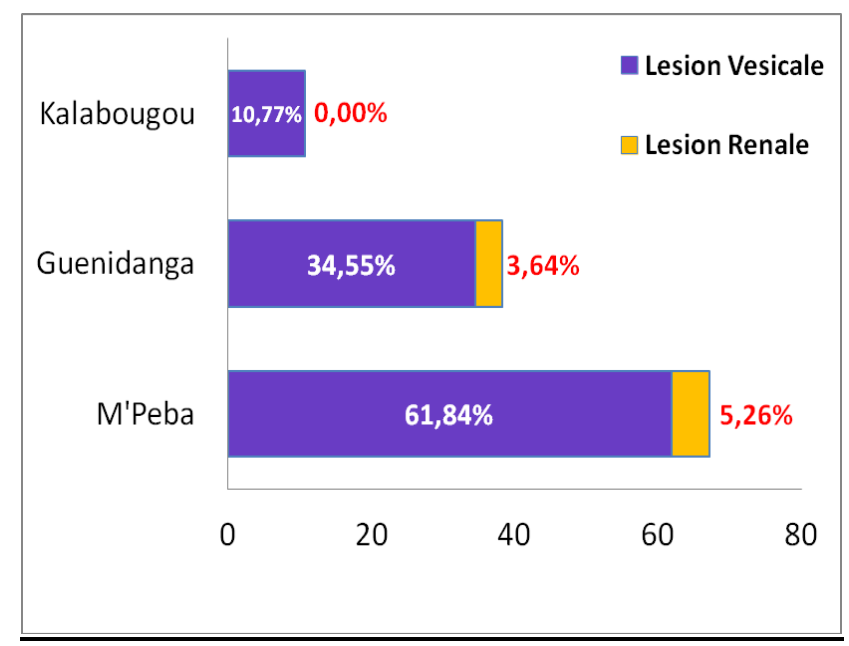

Figure 3 : Prévalence des lésions vésicales et des lésions rénales provoquées par la schistosomiase a S. haematobium dans les villages d'études. $\mathrm{P}<10^{-6}$

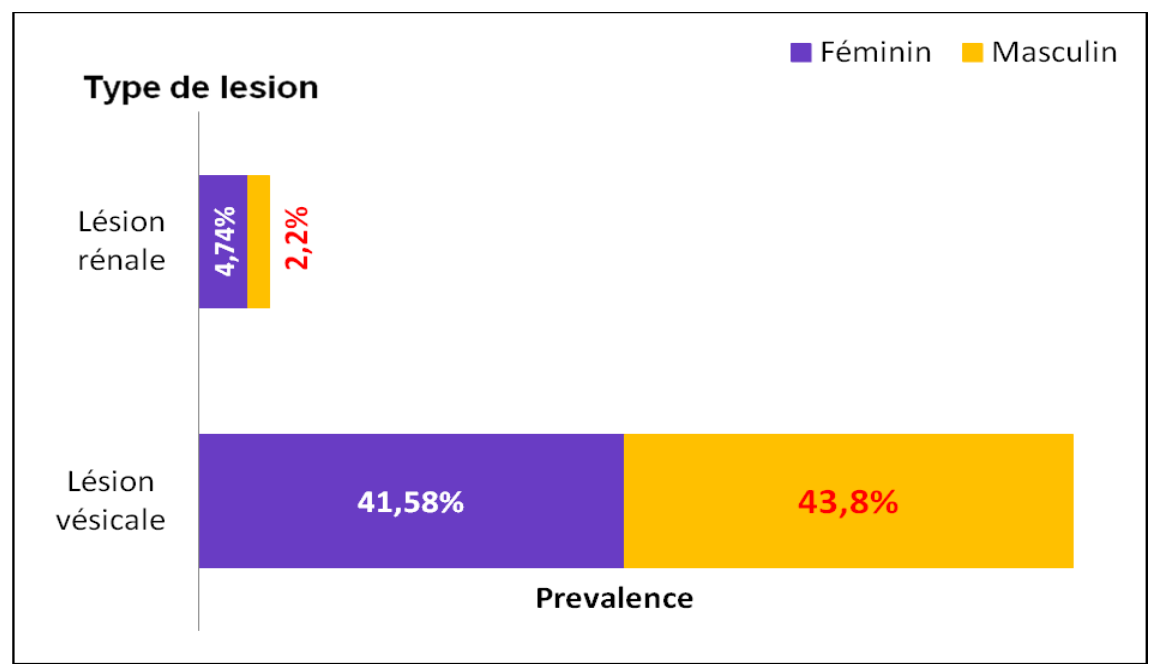

Figure 4: Prévalence des lésions vésicales et des lésions rénales provoquées par la schistosomiase a S. haematobium chez les volontaires en fonction du sexe. $\mathrm{P}=0.226$.

Tableau 1 : Répartition des sujets de l'étude par sexe et par village.

\begin{tabular}{lllllllll}
\hline \multirow{2}{*}{ Sexe } & \multicolumn{2}{l}{\begin{tabular}{l}
\multicolumn{2}{l}{ Villages } \\
Guenidanga
\end{tabular}} & \multicolumn{2}{l}{ Kalabougou } & \multicolumn{2}{l}{ M'peba } & \multicolumn{2}{l}{ Total } \\
\cline { 2 - 10 } & $\mathrm{N}$ & $\%$ & $\mathrm{~N}$ & $\%$ & $\mathrm{~N}$ & $\%$ & $\mathrm{~N}$ & $\%$ \\
\hline Féminin & 69 & 62.7 & 34 & 52.3 & 87 & 57.2 & 190 & 58.10 \\
Masculin & 41 & 37.3 & 31 & 47.7 & 65 & 42.8 & 137 & 41.90 \\
Total & 110 & 100 & 65 & 100 & 152 & 100 & 327 & 100 \\
\hline
\end{tabular}

$\mathrm{P}=0.003 ; \mathrm{N}=$ Nombre de sujets. 
Tableau 2 : Répartition des sujets par tranches d'âge et par village d'étude.

\begin{tabular}{lllllllll}
\hline $\begin{array}{l}\text { Tranche } \\
\text { d'âge } \\
\text { (en année) }\end{array}$ & \multicolumn{2}{l}{$\begin{array}{l}\text { Villages } \\
\text { Guenidanga }\end{array}$} & \multicolumn{2}{c}{ Kalabougou } & \multicolumn{2}{c}{ M'peba } & \multicolumn{2}{c}{ Total } \\
\cline { 2 - 10 } & $\mathrm{N}$ & $\%$ & $\mathrm{~N}$ & $\%$ & $\mathrm{~N}$ & $\%$ & $\mathrm{~N}$ & $\%$ \\
\hline$\leq 5$ & 0 & 0 & 0 & 0 & 60 & 39.48 & 60 & 18.3 \\
] 5-14] & 48 & 43.64 & 30 & 46.15 & 88 & 57.92 & 166 & 50.8 \\
] 14-24] & 15 & 13.64 & 10 & 15.38 & 4 & 2.60 & 29 & 8.9 \\
$\geq 24$ & 47 & 42.72 & 25 & 38.46 & 0 & 0 & 72 & 22.0 \\
TOTAL & 110 & 100 & 65 & 100 & 152 & 100 & 327 & 100 \\
\hline
\end{tabular}

Tableau 3: Parasitémie des sujets de l'étude par sexe et en fonction de l'intensité de leur charge ovulaire en S. haematobium.

\begin{tabular}{lllllll}
\hline \multirow{2}{*}{ Sexe } & & Faible & \multicolumn{3}{c}{$\begin{array}{c}\text { Charge ovulaire } \\
\text { Moyenne }\end{array}$} & \multicolumn{2}{c}{ Elevée } \\
\cline { 2 - 7 } & $\mathrm{N}$ & $\%$ & $\mathrm{~N}$ & $\%$ & $\mathrm{~N}$ & $\%$ \\
\hline Féminin & 79 & 60.31 & 8 & 50 & 20 & 57.14 \\
Masculin & 52 & 39.69 & 8 & 50 & 15 & 42.86 \\
Total & 131 & 100 & 16 & 100 & 35 & 100 \\
& & & & & & \\
\hline
\end{tabular}

$\mathrm{N}=$ nombre de sujets $; \mathrm{p}=0.714$

Tableau 4: Prévalence de l'infection a $S$. haematobium dans les villages n'guenidanga. Kalabougou et m'peba pendant la période d'étude.

\begin{tabular}{|c|c|c|c|}
\hline Villages & Nombre de cas positifs & $\begin{array}{l}\text { Nombre de } \\
\text { dépistes }\end{array}$ & Prévalence \\
\hline GUENIDANGA & 58 & 110 & $52.72 \%$ \\
\hline KALABOUGOU & 16 & 65 & $24.61 \%$ \\
\hline M'PEBA & 110 & 152 & $72.36 \%$ \\
\hline
\end{tabular}

Tableau 5 : Prévalence de l'infection a $S$. haematobium par sexe et par village d'étude.

\begin{tabular}{lllllllll}
\hline \multirow{3}{*}{ Sexes } & \multicolumn{1}{l}{ Villages } & \multicolumn{1}{l}{ Kalabougou } & M'peba & \multicolumn{3}{c}{ Total } & \\
\cline { 2 - 7 } & Guenidanga & & & & \\
& Positif/ $\mathrm{n}$ & $P(\%)$ & Positif/n & $P(\%)$ & Positif/n & $P(\%)$ & Positif/n & $P(\%)$ \\
\hline Féminin & $36 / 69$ & 52.17 & $6 / 34$ & 17.65 & $66 / 87$ & 75.86 & $108 / 190$ & 57.84 \\
Masculin & $22 / 41$ & 53.66 & $10 / 31$ & 32.26 & $44 / 65$ & 67.69 & $76 / 137$ & 55.47 \\
Total & $58 / 110$ & 52.73 & $16 / 65$ & 24.62 & $110 / 152$ & 72.37 & $184 / 327$ & 25.69 \\
\hline
\end{tabular}

$\mathrm{N}=$ nombre de sujets volontaires ; positif $=$ sujets parasites ; $p=$ prévalence $(\mathrm{en} \%) ; \mathrm{p}=0.662$ 


\section{DISCUSSION}

$\mathrm{Au}$ total 327 sujets ont été diagnostiqués dans cette étude. Le nombre de sujets de sexe féminin $(58,10 \%)$ étaient supérieur à celui des sujets de sexe masculin $(41,90 \%)$. Cette tendance était observée dans l'ensemble des trois villages. Cela apparaît être dû au fait que les femmes, pour des raisons de leurs activités ménagères quotidiennes ont plus d'engouement pour le diagnostic de la schistosomiase. Beaucoup d'études ont montré la forte participation des individus de sexe féminin par rapport au sexe masculin , mais aussi une forte participation des enfants d'âge compris entre 3 à 20 ans comme reporté dans les Tableaux 1 et 2 (N'Diaye, 2009; Tchouanguem et al., 2016). Par contre Traoré au cours - d'une étude de co infection Schistosomiase-Paludisme chez des sujets de 4 à 14 ans au Mali a trouvé une prévalence d'infection de $73,80 \%$ chez les garçons et $66,50 \%$ chez les filles (Traore et al., 2007). Une prévalence globale de 56,7\% d'infection par $S$. haematobium a été obtenue dans cette étude, ce qui est diffèrent de 12,6\% obtenu par Adoubryn et al., 2006. Mais une prévalence plus élevée a été rencontrée au Mali par d'autres auteurs comme Kouriba (2004) qui avait abouti à une infection de $100 \%$ chez les enfants de 4 à 7 ans à Bandiagara au cours d'une étude sur l'immunité antibilharzienne et Traoré (2007) avait trouvé $73,90 \%$ d'infection de schistosomes chez des enfants de Dialakoroba au cours d'une étude de co-infection Schistosomiase-Paludisme chez des sujets de 4 à 14. Une forte co-infection SchistosomiasePaludisme avait été aussi trouvée au Nigeria par Adedoja et al. (2015). La mesure de l'intensité de la charge ovulaire due à $S$. haematobium a été faite selon le barème de l'OMS. Le rapport de sexe (Féminin/Masculin) par rapport à l'intensité de la charge ovulaire est de $79 / 52 ; 8 / 8$ et $20 / 15$ respectivement pour les sujets ayant une charge ovulaire faible, moyenne et élevée (Tableau 3). Les tests statistiques ont montré qu'il n'y a pas de différence significative entre les sujets de sexe différent par rapport à l'intensité de la charge ovulaire quand il s'agit d'une même catégorie de charge ovulaire ( $P \geq 0,714$, Tableau 3) (Traore et al., 2007). Dawet et al. (2012) ont aussi trouvé dans deux localités du Nigeria que le taux d'infection à S. haematobium ne variait ni par sexe, ni par groupe d'âge ni par la nature de l'occupation, mais il y avait une relation significative entre la source d'approvisionnement en eau et le niveau d'infection à $S$. haematobium. Par contre Zongo au Burkina Faso, Diakité et Traoré au Mali ont montré que les garçons avaient souvent une infection plus élevée par rapport aux filles (Coulibaly, 2001; Zongo et al., 2012). A Yamoussoukro en Côte d'Ivoire également, Orsot et al. (2018) ont trouvé que la prévalence de l'infection de la bilharziose est en relation avec l'âge et le sexe.

La répartition des sujets par tranche d'âge a montré que 60 sujets étaient âgés d'au plus 5 ans ; 166 individus étaient âgés entre 514 ans; 29 individus avaient l'âge compris entre 14 - 24 ans; 72 sujets étaient âgés de 24 ans. Il apparait clairement que les sujets les plus vulnérables et les plus susceptibles à l'infection des schistosomes sont ceux âgés de 5 à 14 ans. A partir de 24 ans, la prise de conscience et les différentes sensibilisations peuvent contribuer à réduire le risque d'infection chez les sujets de plus de 24 ans. Ce résultat corrobore ceux d'autres auteurs (Ibikounlé et al., 2013) qui avaient aussi trouvé que les sujets d'âge inférieur à 14 ans étaient plus parasités que ceux d'âge supérieur à 14 ans.

La comparaison entre les trois sites d'étude a montré que la prévalence de l'infection à $S$. haematobium dans le village de M'Pèba (72,36\%) était nettement supérieure à celles des villages de Guénidanga $(52,61 \%)$ et de Kalabougou $(24,4 \%) ; \mathrm{P}<10^{-6}$ (Tableau 4). Ces taux de prévalence de $S$. haematobium sont nettement supérieurs à ceux obtenus par Zongo et al. (2016) dans deux régions du Burkina Faso soient $4 \%$ et $0,6 \%$ avec une moyenne de $2,3 \%$ (95\% CI: $1,5 \%-3,1 \%)$. Pour tous les villages 
confondus, la prévalence de l'infection à $S$. haematobium entre sujets de sexe différent n'était pas significativement différente. La prévalence de l'infection au sein du groupe des sujets de sexe féminin $(57,84 \%)$ était comparable à celle au sein du groupe des sujets de sexe masculin soit $55,73 \%$ (Tableau 5). Ce résultat corrobore celui de Garcia et al., 2013, qui ont trouvé qu'au Cameroun, les hommes et les femmes d'un périmètre hydroaménagé ont un taux d'infestation similaire de $S$. haematobium, et que le niveau de transmission va de pair avec la pratique des activités hydro-anthropiques quel que soit la saison avec l'absence des latrines. La hausse de l'infection à M'Pèba pourrait être due au fait que tous les sujets représentant ce village avaient un jeune âge donc plus vulnérables. Car l'analyse de la charge ovulaire par tranche d'âge a montré la nette prédominance des sujets de 5 à 14 ans dans les différents groupes de sujet à charge ovulaire faible, moyenne et élevée. Le jeune âge apparaît donc comme un facteur d'exposition à la forte charge ovulaire. L'analyse de corrélation montre aussi qu'il y a une relation inversement proportionnelle entre l'âge des sujets et l'intensité de la charge ovulaire ce qui peut avoir une influence sur la morbidité comme évoqué par certains travaux d'autres antérieurs (Engels, 2002; 2003). Dans le même ordre d'idée, certains auteurs ont eu une intensité de forte charge ovulaire $(13,8 \%)$ chez les enfants d'âges scolaire de 7 à 14 ans dans la région de Ségou au Mali. La vulnérabilité des sujets jeunes $(5-24)$ à la charge ovulaire élevée peut s'expliquer par leur contact fréquent avec l'eau pour diverses raisons, entre autres : baignade, jeux, pêches lucratives, justifiant ainsi les facteurs de risque de portée épidémiologique (N'Gbesso et al., 2017).

Comme déjà rapporté par des études antérieures, l'infestation par S. haematobium entraine de très nombreux cas de maladie. Jusqu'à $50-70 \%$ des sujets parasités d'une localité ou la maladie est endémique présentent des troubles urinaires. Ainsi notre étude a montré des cas de complication ayant abouti à des lésions. La lésion vésicale était rencontrée dans toutes les tranches d'âges, alors que la lésion rénale n'a été rencontrée que chez les sujets âgés d'au plus 20 ans (Figure 3). L'importance des lésions est inversement proportionnelle à l'âge des sujets. En d'autres termes, les sujets de 5-14 ans avec $61,94 \%$ sont plus exposés au lésions par rapport à ceux de 14 à 24 ans $(48,91 \%)$, et ceux ayant plus de 24 ans. Par contre les sujets ayant présenté plus de lésion rénale sont ceux âgés de 14 à 24 ans. Une comparaison entre sujets féminins et masculins des prévalences de la lésion aussi bien vésicale $(\mathrm{P}=0,68)$ que rénale $(\mathrm{P}=0,226)$ a montré qu'il n'y a pas de différence significative liée au sexe lorsqu'il s'agit du même type de lésion (Figure 4). Les affections urogénitales dues à la complication de la schistosomiase ont été observées par beaucoup d'autres auteurs à travers le monde depuis longtemps (Adedoja et al., 2015) mais le problème demeure encore de nos jours avec souvent un impact économique important (Moné, 2010) ; d'où l'importance d'une investigation plus approfondie pour prévenir ou limiter l'ampleur (Bosier, 2001).

\section{Conclusion}

La réussite des campagnes de lutte contre les schistosomiases est supposée éradiquer ou réduire considérablement la prévalence des infections dues aux schistosomes. Mais les résultats obtenus au cours de cette étude montrent que malgré l'existence des campagnes de traitement de masse dans les trois villages, la schistosomiase demeure toujours un problème de santé publique. La persistance de cette parasitose dans ces villages suscite deux hypothèses majeures :

(i) Il y a apparition probable d'une résistance à la molécule utilisée pendant les campagnes.

(ii) Augmentation du vecteur (mollusque) impliquant la réinfection rapide dans les sites d'études. 
Quelle que soit l'hypothèse, nous suggérons plus de vigilance à l'exécution des différentes campagnes de lutte et recommandons que les deux hypothèses citées ci-dessus soient vérifiées par des études scientifiques ultérieures.

\section{CONFLIT D'INTERETS}

Les auteurs déclarent qu'ils n'ont aucun conflit d'intérêts.

\section{CONTRIBUTIONS DES AUTEURS}

La conception du protocole d'étude a été faite par BS, BT, ASY et MS, le travail de terrain y comprise la collecte des données a été faite par BT, BS. La conception de la base de données, le nettoyage, l'analyse et l'interprétation des donnée a été faite par ASY. Le premier manuscrit a été écrit par ASY et BT, tous les auteurs ont lu et accepté la dernière version.

\section{REMERCIEMENTS}

Un remerciement sincère au personnel de l'INRSP (Institut National de Recherche en Santé Publique) et aux membres du Laboratoire d'Entomologie Parasitologie de la FST-USTTB de Bamako, MALI.

\section{RÉFÉRENCES}

Adedoja AA, Akanbi AA, Oshodi AJ. 2015. Effect of artemether-lumefantrine treatment of falciparum malaria on urogenital schistosomiasis in co-infected School Aged Children in North Central of Nigeria. Int. J. Biol. Chem. Sci., 9(1): 134-140.

DOI: http://dx.doi.org/10.4314/ijbcs.v9i1.13.

Adoubryn KD, Ouhon J, Yapo CG, Assoumou EY, Ago KML, Assoumou A. 2006. Profil épidémiologique des schistosomoses chez les enfants d'âge scolaire dans la région de l'Agnéby (sudest de la Côte-d'Ivoire). Bull Soc Pathol Exot., 99(1): 28-31. DOI: 10.3185/pathexo2644

Aubry P. 2016. Schistosomoses ou bilharzioses.

Actualités. http:/medecinetropicale.free.fr/cours/schi stosomes.htm.

Bosier P. 2001. Schistosome à Schistosoma mansoni : évaluation échographique de la morbidité et de son évolution après administration de praziquantel : Etude à Madagascar. Thèse de doctorat en Sciences biologiques et médicales à Bordeaux 2, Épidémiologie et Intervention en santé Publique.

Chippaux JP. 2000. Control of schistosomiasis: realities and futurology. Médecine Tropicale, 60: 54-55.

Chitsulo L, Loverde P, Engels D. 2004. Schistosomiasis. Nat. Rev. Microbiol., 2: 1213.

Clements AC, Garba A, Sacko M, Touré S, Dembelé R, Landouré A, Bosque Oliva E, Gabrielli A, Fenwick FA. 2008. Cartographie de la probabilité de schistosomiase et de l'incertitude associée en Afrique de l'Ouest. Ouest. Afrique Emerg. Infecter. Dis., 14: 16201632.

Coulibaly H. 2001. L'Evaluations épidémiologiques, étude clinique et enquête sur les connaissances, attitudes et pratiques de la population en matière de bilharziose en milieu périurbain du district de Bamako (djicoroni para). Thèse de médecine, Bamako, 74p.

Dawet A, Yakubu DP, Longmut R, Benjamin CB, Daburum YH, Nannim N. 2012. Prevalence and intensity of Schistosoma haematobium among residents of Gwong and Kabong in Jos North Local Government Area, Plateau State, Nigeria. Int. J. Biol. Chem. Sci., 6(4): 1557-1565.

DOI: http://dx.doi.org/10.4314/ijbcs.v6i4.15

Engels D, Chitsulo L, Montresor A, Savioli L. 2002. The global epidemiological situation of schistosomiasis and new approaches to control and research. Acta Tropica, 82:139-146.

Engel D. 2003. Quantification of clinical mordibity assciated with schistosome 
infection in sub-saharan africa. Acta Tropica, 86: 125-139.

Garcia S, Njan NMA, Tchuenguem FFN. 2013. Effets des aménagements hydrauliques sur la transmission de Schistosoma haematobium dans la plaine $\mathrm{du}$ Logone, région de l'Extrême-Nord Cameroun. Int. J. Biol. Chem. Sci., 7(4): 1629-1639.

DOI:

http://dx.doi.org/10.4314/ijbcs.v7i4.19

Ibikounlé M, Satoguina J, Fachinan R, Tokplonou L, Batcho W, Kindé-Gazard D, Mouahid G, Moné H, Massougbodji A, Courtin D. 2013. Épidémiologie de la bilharziose urinaire et des geohelminthiases chez les jeunes scolaires des zones lacustres de la commune de So-Ava. Sud-Bénin Journal of Applied Biosciences, 70: 5632- 5639.

Kouriba B. 2004. Analyse des facteurs qui modulent l'immunité antibilharzienne dans une population Dogon infectée par S. harmatobium. Thèse de Doctorat de l'université de la Méditerranée, AIXMarseille II.

Moné H, Ibikounlé M, Massougbodji A, Mouahid G. 2010. Human schistosomiasis in the Economic Community of West African States (ECOWAS): epidemiology and control. Advances in Parasitology, 71: 33-91.

Moné H, Minguez S, Ibikounlé M, Allienne JF, Massougbodji A, Mouahid G. 2012. Natural Interactions between $S$. haematobium and $\mathrm{S}$. guineensis in the Republic of Benin. The Scientific World Journal, ID 793420: 8p. DOI: http://dx.doi.org/10.1100/2012/793420

Molyneux DH, Hotez PJ, Fenwick A. 2005. "Rapid impact intervention": how a policy of integrated control for Africa's neglected tropical diseases. PLoS Med ., 2(11): e336. DOI: https://doi.org/10.1371/journal.pmed.002 0336

N'Diaye F. 2009. Impact du traitement de masse au praziquantel sur la morbidité due aux shistosomiases dans le district de Bamako et le cercle de Kati. Thèse de Médecine (DIPLOME D’ETAT), FMOS-USTTB, point G Bamako.

N'Gbesso NJP, N'Guessan NA, Assaré RK, Orsoti NM, N'Dri K, Yapi A. 2017. Epidemiology of schistosomiasis in Ahoué, southern Côte d'Ivoire. International Journal of Innovation and Applied Studies, 21: 378-387.

N'Goran EK, N' Guessan A, Muller I, Zamble K, Lohourignon KJ, Traore M, Sosthene BA, Lengeler C, Tanner M. 2001. Reinfection with Schistosoma haematobium following school-based chemotherapy with praziquantel in four highly endemic village in Cote d'Ivoire. Trop. Med. Int. health, 6. DOI: https://doi.org/10.1046/j.13653156.2001.00785.x

OMS. 2010. Global trends in schistosomiasis control.

OMS.

www.who.int/bulletin/86/10/08058669/en

OMS. 2016. Aide-mémoire N 115. OMS. hpp://www.who.int/mediacentre/factshee $\mathrm{t} / \mathrm{fs} 115 / \mathrm{fr}$

Orsot MN, Meite A, N'guessan NA, Dje NN, Ouattara M, Coulibaly JT, Assare RK, Diakité NR, Mama AD, Dje LMA, N'Goran EK. 2018. Detailed distribution of schistosomiasis and soil-transmitted helminthiasis among schoolchildren in the Bélier and Marahoué regions, central Côte d'Ivoire: a tool for an efficient control. Int. J. Biol. Chem. Sci., 12(4): 1532-1542.

DOI: https://dx.doi.org/10.4314/ijbcs.v12i4.1

Stecher wc, Madsen H, Shona W, Sacko M, Wejse C, Keita AD, Landouré A, Traoré M, Kallestrup P, Petersen E, Vennervald B. 2017. Organomegaly in Mali before and after praziquantel treatment. A possible association with Schistosoma haematobium. Heliyon, 3 (11): e00440. DOI: https://doi.org/10.1016/j.heliyon.2017.e 0440 
Steinman P, Keiser J, Bos R, Tanner M, Utzinger J. 2006. Schistosomiasis and water resources development: systematic review, meta-analysis and estimates of people at risk. Lancet Infect. Dis., 6: 411-425.

DOI: [10.1016/j.heliyon.2017.e00440]

Tandina F, Doumbo SN, Kone AK, Guindo D, Goita S, Sissoko M, Konate S,. Dabo A, Doumbo OK. 2016. Épidémiologie des schistosomoses dans le village périurbain de Sotuba, dix années après la mise à échelle du traitement de masse au Mali. Medecine et Sante Tropicales, 26: 51-56. DOI:10.1684/mst.2015.0515.

Tangara M. 2002. Aspects chirurgicaux des séquelles de la bilharziose urinaire. Thèse de Médecine, Bamako, 80p.

Tchouanguem HN, Fouelifack FY, Keugoung B, Fouelifa LD, Moyou RS. 2016. Situation actuelle de la schistosomiase dans l'aire de santé de Santchou. (District de santé de Santchou. Région de l'Ouest-Cameroun). Pan African Medical Journal, $\quad$ 24: 137 DOI: 10.11604/pamj.2016.24.137.8778.

Traore M, Landouré A, Diarra A, Kante B, Sacko M, Coulibaly G, Sangho A, Simaga SY. 2007. La diversité écologique et épidémiologique des schistosomiases au Mali: implication pour un programme de contrôle. Mali Medical. Tome XXII, 3: 22-28.

WHO. 2011. Rapport du comité O. M. S. d'experts sur la lutte contre les maladies tropicales négligées. WHO. www.who.int/iris/handle/10665/42588.

Zongo D, Kabre BG, Dayeri D, Savadogo B, Poda JN. 2012. Comparative study of schistosomiasis transmission (urinary and intestinal forms) at 10 sites in Burkina Faso (in sub-Saharan Africa). Med Sante Trop., 22: 323-329.

Zongo D, Bagayani $\mathrm{M}$, Tiendrebeogo $\mathrm{S}$, Drabo F, Ouedraogo H, Savadogo B, Bamba I, Yago-Vienne F, Zhang Y, Poda JN. 2016. Int. J. Biol. Chem. Sci., 10(4): $\quad$ 1525-1533. DOI: http://dx.doi.org/10.4314/ijbcs.v10i4.6

Yapi GY, Touré M, Sar MD, Abo N, Diabaté S. 2017. The impact of irrigated rice on the transmission of schistosomiasis and geohelminthiasis in Niakaramandougou. Côte d'Ivoire. Int. J. Biol. Chem. Sci., 11(4): 1400-1412. DOI: http://dx.doi.org/10.4314/ijbcs.v11i4.1 